\title{
Electronic structure of a neutral oxygen vacancy in $\mathrm{SrTiO}_{3}$
}

\author{
Davide Ricci, Giuseppe Bano, and Gianfranco Pacchioni* \\ Dipartimento di Scienza dei Materiali, Università di Milano-Bicocca, and Istituto Nazionale per la Fisica della Materia, \\ via R. Cozzi 53, I-20125 Milano, Italy
}

Francesc Illas

Departament de Química Física i Centre de Recerca en Química Teòrica, Universitat de Barcelona i Parc Científic de Barcelona, C/ Martí i Franquès 1, E-08028 Barcelona, Spain

(Received 29 July 2003; published 11 December 2003)

\begin{abstract}
The electronic structure of an isolated oxygen vacancy in $\mathrm{SrTiO}_{3}$ has been investigated with a variety of $a b$ initio quantum mechanical approaches. In particular we compared pure density functional theory (DFT) approaches with the Hartree-Fock method, and with hybrid methods where the exchange term is treated in a mixed way. Both local cluster models and periodic calculations with large supercells containing up to 80 atoms have been performed. Both diamagnetic (singlet state) and paramagnetic (triplet state) solutions have been considered. We found that the formation of an $\mathrm{O}$ vacancy is accompanied by the transfer of two electrons to the $3 d\left(z^{2}\right)$ orbitals of the two Ti atoms along the Ti-Vac-Ti axis. The two electrons are spin coupled and the ground state is diamagnetic. New states associated with the defect center appear in the gap just below the conduction band edge. The formation energy computed with respect to an isolated oxygen atom in the triplet state is $9.4 \mathrm{eV}$.
\end{abstract}

DOI: $10.1103 /$ PhysRevB.68.224105

PACS number(s): 61.72.Bb, 31.15.Ar

\section{INTRODUCTION}

Point defects often determine the performance and the chemical stability and activity of oxide materials. ${ }^{1}$ Hence, the study of point defects is of fundamental importance in the design of microelectronic devices, optical fibres, sensors, transparent conductors, ferroelectrics, catalysts, etc. In the past decades the knowledge about defects has grown to the point that their nature and concentration is controlled in a fine way, leading to the birth of a new discipline, defect engineering. Defect engineering is aimed at manipulating the nature and the concentration of defects in a material so as to tune its properties in a desired manner or to generate completely new and unexpected behaviors. For instance, by changing the defect concentration one can turn a colorless insulator into a black material with metallic conductivity or an ordinary copper oxide into a high temperature superconductor. This is for instance the case of $\mathrm{SrTiO}_{3}$, a band insulator with a gap of $3.2 \mathrm{eV}^{2}$ well known as a ferroelectric material. By doping $\mathrm{SrTiO}_{3}$ with $\mathrm{La}$ atoms and/or by creation of oxygen vacancies the system undergoes an insulator-tometal transition and even becomes a superconductor at very low temperatures $\left(T_{c}<0.3 \mathrm{~K}\right){ }^{3}$ It is not surprising that the nature of the oxygen vacancy in $\mathrm{SrTiO}_{3}$ and in general in perovskites has attracted the attention of theorists in the past. $^{4-13}$

An essential contribution to the knowledge of the geometric and electronic structure of intrinsic and extrinsic defects comes from theoretical approaches. The rudiments of these theories has been laid in the 1930s with the methods of molecular orbital theory, ${ }^{14}$ the theories of Thomas and Fermi, ${ }^{15}$ and Mott and Littleton. ${ }^{16}$ The advent of modern density functional theory (DFT) based on the Kohn-Sham equations ${ }^{17}$ and the Hellman-Feynman theorem has established a completely new basis to elucidate the nature of defects in solids.
Nearly all ab initio studies reported on the electronic structure of an $\mathrm{O}$ vacancy in perovskites are based on DFT. ${ }^{6,10,11,13}$

However, there are some aspects of the nature of point defects, in particular related to their magnetic behavior, that are difficult to treat at the DFT level. ${ }^{18-20}$ One recent and illustrative example is that of the spin localization in an $\mathrm{Al}$ substitutional impurity in $\mathrm{SiO}_{2}$. Because of the selfinteraction problem, DFT calculations predict that the hole associated with a neutral $\left[\mathrm{AlO}_{4}\right]^{0}$ center is delocalized over four oxygen atoms, contrary to accurate EPR experiments ${ }^{21}$ and Hartree-Fock calculations that indicate full localization of the hole on a single bridging oxygen. ${ }^{22} \mathrm{~A}$ similar problem can be found every time the electrons associated with a defect can be localized or delocalized on a given state of the system.

Depending on the material, the structure and properties of oxygen vacancies can vary substantially. In $\mathrm{MgO}$, a highly ionic compound, a missing $\mathrm{O}$ atom results in two trapped electrons localized in the cavity; the driving force for the localization is the Madelung potential of the ionic crystal. The removal of a neutral $\mathrm{O}$ atom from $\mathrm{MgO}$ results in a very small local relaxation (the crystalline potential is only moderately perturbed and the distances around the vacancy change by less than $1 \%$ ). The defect center gives rise to typical excitations in the visible and UV regions of the spectrum and is known as F center, from Farbe, the German word for color. The situation is completely different in $\mathrm{SiO}_{2}$, a solid characterized by covalent polar bonds. In $\mathrm{SiO}_{2}$ the removal of an $\mathrm{O}$ atom from $a \equiv \mathrm{Si}-\mathrm{O}-\mathrm{Si} \equiv$ linkage (neutral oxygen vacancy) results in two $\mathrm{Si}$ dangling bonds, $\equiv \mathrm{Si}$, which recombine to form $a \equiv \mathrm{Si}-\mathrm{Si} \equiv$ covalent bond with two electrons occupying a localized state with $\mathrm{Si}$-Si bonding character. ${ }^{23}$ The process is accompanied by a strong geometrical relaxation around the defect. The associated optical 
transition involves localized excitations from a bonding to an antibonding state in the gap. ${ }^{23}$ The nature of the oxygen vacancy in the two oxides, therefore, is completely different as the result of the different electronic structure and bonding in the two materials, highly ionic in $\mathrm{MgO}$, covalent polar in $\mathrm{SiO}_{2}$. Less investigated are transition metal oxides with a degree of ionicity intermediate between fully ionic and fully covalent. Here an additional complication comes from the presence of $d$ orbitals on the metal ion. This opens in fact new possibilities to redistribute the electrons involved in the bonding with the oxygen atom which has been removed. A characteristic of most of the transition metal oxides is the possibility to change the oxidation state of the metal. In $\mathrm{TiO}_{2}$, a model system for transition metal oxides, the electrons of an oxygen vacancy are neither trapped in the cavity nor lead to the formation of a metal-metal bond. Rather, they are transferred to the empty $3 d$ levels at the bottom of the conduction band belonging to the adjacent $\mathrm{Ti}$ atoms. ${ }^{24,25,26}$ Since the $3 d$ states are rather localized, this corresponds to a change of the formal oxidation state, from $\mathrm{Ti}^{+4}$ to $\mathrm{Ti}^{+3}$. The resulting ground state is magnetic (triplet). ${ }^{24,25}$ The three examples illustrated here, $\mathrm{MgO}, \mathrm{SiO}_{2}$, and $\mathrm{TiO}_{2}$, are paradigmatic of the great variety of behaviors of oxygen vacancies in simple binary oxides.

The complexity of the situation reflects also in the analysis of the literature data on the oxygen vacancy in $\mathrm{SrTiO}_{3}$. Strontium titanate has a cubic structure and comprises highly ionic bonds ( $\mathrm{Sr}-\mathrm{O})$ and more covalent bonds (Ti-O). Thus, $\mathrm{SrTiO}_{3}$ presents the characteristics of both an ionic oxide and a transition metal oxide, and some ambiguity exists about the nature of an oxygen vacancy. While most studies suggest an involvement of the Ti $3 d$ empty states in the formation of the oxygen vacancy, ${ }^{7,10,11}$ some point to a partial or total localization of the electrons in the cavity; ${ }^{4,12}$ some studies show the formation of impurity levels in the gap of the material near the conduction band edge ${ }^{5}$ while others found a shift of the Fermi level inside the conduction band by creating $\mathrm{O}$ vacancies. ${ }^{6,7}$

Scope of this paper is to provide a general description of the structure of an oxygen vacancy in $\mathrm{SrTiO}_{3}$ based on the two most commonly used solid state physics approaches (local cluster models and periodic band structure methods), different DFT methods based on various expressions of the exchange-correlation functionals, as well as on pure HartreeFock calculations. Particular attention will be given to the possible existence of magnetic ground states and to spin localization phenomena.

\section{DETAILS OF CALCULATIONS}

$\mathrm{SrTiO}_{3}$ at room temperature has the cubic structure of perovkites with a cubic cell of $3.9051 \AA$, while below $105 \mathrm{~K}$ is present in a tetragonal phase. Here we considered the cubic phase. For the description of an $\mathrm{O}$ vacancy in $\mathrm{SrTiO}_{3}$ we used two different approaches, one based on cluster models and the other on periodic supercell band structure calculations. In the following we describe the main features of the two approaches.
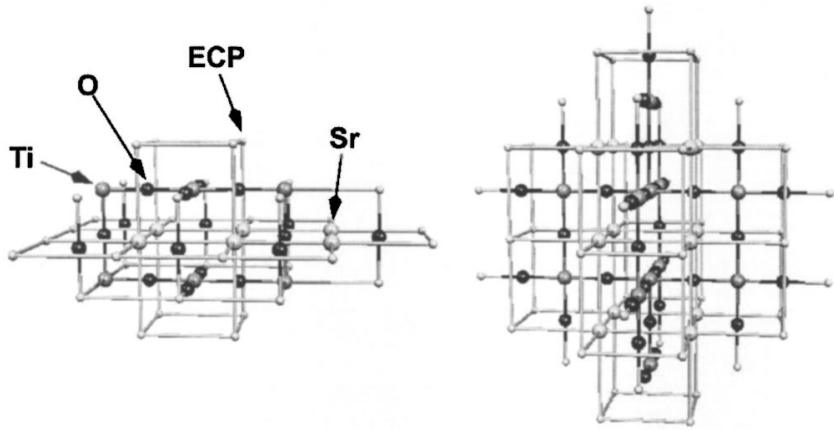

FIG. 1. Cluster models of an oxygen vacancy in bulk $\mathrm{SrTiO}_{3}$. Left: $\mathrm{Sr}_{6} \mathrm{Ti}_{6} \mathrm{O}_{18}$. Right: $\mathrm{Sr}_{12} \mathrm{Ti}_{12} \mathrm{O}_{36}$. The clusters are surrounded by $\mathrm{Ti}$ and $\mathrm{Sr}$ atoms treated with an ECP and by a large array of point charges (not shown). The missing $\mathrm{O}$ atom at the center has been removed to form an $\mathrm{O}$ vacancy.

\section{A. Cluster calculations}

$\mathrm{SrTiO}_{3}$ is a largely ionic crystal, and the description of an oxygen vacancy in the material using a finite number of atoms requires the inclusion of the effect of the long-range Madelung potential of the extended crystal. This has been done following a widely used approach. A cluster of ions is embedded into a large matrix of classical ions represented by point charges (PC) of nominal values, i.e., +2 for $\mathrm{Sr},+4$ for $\mathrm{Ti}$, and -2 for $\mathrm{O}$. To this end, an array of 6655 PC's has been constructed. With this grid of PC's the Madelung field, -1.7487 a.u., is well converged and is reasonably homogeneous in a region of about $40 \AA$ centered around the vacancy.

The local region where the defect is created has been treated quantum-mechanically $(\mathrm{QM})$. In particular, we used two stoichiometric QM clusters, $\mathrm{Sr}_{6} \mathrm{Ti}_{6} \mathrm{O}_{18}$ and $\mathrm{Sr}_{12} \mathrm{Ti}_{12} \mathrm{O}_{36}$, Fig. 1. Stoichiometry is essential to avoid the artificial assignment of extra charges to the QM cluster in order to respect the formal oxidation number of the constituent ions. An interface between the QM cluster and the classical ions is needed in order to prevent an artificial spreading of electronic states outside the QM cluster. The interface includes $\mathrm{Sr}$ and $\mathrm{Ti}$ atoms only; these are represented by semilocal effective core potentials (ECP). ${ }^{27}$ The interface atoms interact quantum mechanically with the atoms in the QM cluster and classically with PC's in the external region. The ECP atoms do not have basis functions associated and provide a simple representation of the finite size of the ion core.

The wave function of the QM cluster has been constructed by means of atomic Gaussian type orbital basis sets. We used two sets of basis functions that we denote in the following as basis set A and B, respectively. In basis set A the $\mathrm{O}$ atoms are described with the all electron $6-31 \mathrm{G}$ basis. ${ }^{28}$ The Ti atoms are treated with a large core ECP which includes in the valence the $3 d^{2} 4 s^{2}$ electrons only; the basis set is of double-zeta (DZ) quality, $(3 s 2 p 5 d / 2 s 2 p 2 d) .{ }^{29}$ For the $\mathrm{Sr}$ atoms we used a large core ECP which includes in the valence only the $5 s^{2}$ electrons; the DZ basis set is $(3 s 2 p / 2 s 2 p) .{ }^{29} \mathrm{In}$ the more accurate basis set $\mathrm{B}$ the $\mathrm{O}$ atoms are treated with the all electron $6-31+\mathrm{G}^{*}$ basis (including both diffuse and $d$ polarization functions). The Ti atoms are described with a small core ECP which includes in the va- 
lence the $3 s^{2} 3 p^{6} 3 d^{2} 4 s^{2}$ electrons; the corresponding basis set is $(8 s 5 p 5 d / 3 s 3 p 3 d) .{ }^{29}$ For the $\mathrm{Sr}$ atoms we used an ECP which includes in the valence the $4 s^{2} 4 p^{6} 5 s^{2}$ electrons and a $(8 s 6 p / 3 s 3 p)$ basis set. ${ }^{29}$

A problem in the study of vacancies with atomic basis functions is that these are associated with the atomic nuclei. When an atom is removed, the space is not filled by basis functions. A way to circumvent the problem is to place "ghost" atomic orbitals in the cavity. ${ }^{30}$ In particular, we left the functions associated with the $\mathrm{O}$ atom in the center of the vacancy. This provides an higher flexibility to the basis set for the description of the localization (if any) of the trapped electrons in the vacancy.

The calculations were carried using two different theoretical approaches. Hartree-Fock (HF) calculations have been performed in the spirit of determining the wave function of the system. The HF method offers the advantage of an exact treatment of the nonlocal exchange interactions and of the spin states, but suffers from the limitation that correlation effects are completely neglected. Previous experience has shown that the HF method tends to produce solids with a large charge separation (strong ionic contributions), a wide band gap (2-3 times larger than in the experiment), and strongly localized spin states. However, there are examples where the HF method provides the correct physical picture of the spin distribution in a defect center, at variance with the DFT methods which give delocalized states. ${ }^{22}$

The HF calculations have been compared to the DFT approach. Here we used the gradient corrected Becke's three parameters hybrid exchange functiona ${ }^{31}$ in combination with the correlation functional of Lee, Yang, and Parr $5^{32}$ (B3LYP). This method offers the best results in terms of bond strengths in molecules and solids, and in general provides a very accurate description of several properties at the molecular level. ${ }^{33}$ It also provides a reasonable description of the band gap in ionic solids. ${ }^{20,34}$

Most of the calculations have been done without optimizing the cluster geometry. LMTO calculations on $\mathrm{KNbO}_{3}$ have shown an energy gain of $0.5 \mathrm{eV}$ by relaxing the structure accompanied by a partial electron density redistribution between the $\mathrm{O}$ vacancy and the nearest two $\mathrm{Nb}$ atoms. ${ }^{35,36}$ On the other hand, previous LDA calculations have shown that the atomic relaxation around the vacancy consists in a small shifting of the neighboring atoms away from the vacancy site. ${ }^{13}$ It is also known experimentally that the crystal structure and lattice parameters do not vary significantly even for small dopings of $\mathrm{SrTiO}_{3} .{ }^{37}$ The small relaxation around this point defect is confirmed by a partial optimization of the structure with the oxygen vacancy with the $\mathrm{Sr}_{12} \mathrm{Ti}_{12} \mathrm{O}_{36}$ cluster which shows a small displacement of the Ti ions nearest to the vacancy by $0.1 \AA$ and away from it. Therefore, in the following, only ideal bulk structures are considered.

The cluster calculations have been performed using the Gaussian98 program package. ${ }^{38}$

\section{B. Periodic calculations}

The study of regular and defective $\mathrm{SrTiO}_{3}$ has been performed also by means of a periodic supercell approach. We
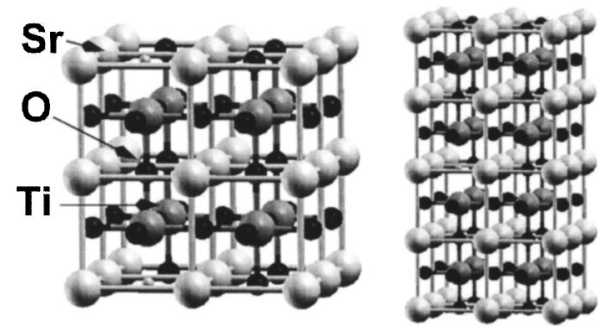

FIG. 2. Supercells used for the periodic calculations. Left: 40 atoms supercell; the minimum distance between two vacancies is $7.86 \AA$; right: 80 atoms supercell; the minimum distance between two vacancies along the $z$ axis is $15.72 \AA$. A small sphere indicates the position of the missing $\mathrm{O}$ atom.

first considered the properties of the ideal $P m 3 m$ lattice. The calculations were performed using five atoms per unit cell, $\mathrm{Sr}, \mathrm{Ti}$, and three $\mathrm{O}$ atoms.

Large supercells of 40 and 80 atoms have been used to simulate an isolated oxygen vacancy, Fig. 2. This corresponds to stoichiometries of the material $\mathrm{SrTiO}_{2.875}$ and $\mathrm{SrTiO}_{2.9375}$, or $\mathrm{SrTiO}_{3-\delta}$, with $\delta=0.125$ and $\delta=0.0625$, respectively. The electronic structure has been determined for both diamagnetic closed shell (singlet) and spin polarized open shell (triplet) solutions. The states considered in unrestricted-HF or spin polarized DFT are not pure triplet states, but the spin contamination is very small. A recent study on a $\mathrm{Fe}$ impurity in $\mathrm{SrTiO}_{3}$ has shown that defectdefect interactions can be quite considerable even for supercells containing 160 atoms, in particular at the DFT level. ${ }^{39}$ In our models the distance between two vacancies along the $z$ axis is $7.86 \AA$ and $15.72 \AA$, respectively, for 40 - and 80 atoms supercells. Going from the small to the large cell we do not observe major changes in the defect electronic structure (atomic charges, defect bandwidth, etc.). Netherveless, it is clear that even at these dimensions the tails of the wave function can extend beyond the limits of the supercell. This can affect to some extent the computed quantities. On the other hand, the focus here is on the qualitative nature of the ground state in an oxygen vacancy in $\mathrm{SrTiO}_{3}$. The limit of infinite dilution is represented by the cluster model (see previous paragraph) where no interaction among defects is present. From this point of view, the comparison of supercells of medium size and local cluster models should provide a firm basis for our conclusions.

The supercell calculations have been performed using a massive parallel version of the CRYSTAL package, ${ }^{40}$ a periodic program using atomic Gaussian type orbital basis sets. The basis set is close (although not identical) to that used in the cluster calculations, making a direct comparison of cluster and periodic results possible. The $\mathrm{Sr}$ ions have been described with a small core ECP, ${ }^{29}$ the $\mathrm{Sr}$ basis set has been taken from Ref. 41. The Ti atoms have been described at the all electron level using a 86-411(d31)G basis set optimized on $\mathrm{TiO}_{2} ;{ }^{42}$ the $\mathrm{O}$ basis set, $8-411 \mathrm{G}$, is also of all electron type and has been used in several studies on oxide crystals. ${ }^{41}$

The Crystal calculations have been done at the HF and DFT levels using $6 \mathrm{~K}$ points. The LDA, B3LYP and PW91 expressions of the exchange-correlation functionals have 
TABLE I. Computed properties of bulk $\mathrm{SrTiO}_{3}$ with various approaches.

\begin{tabular}{ccccc}
\hline \hline Method & $a_{0}, \AA$ & Bulk mod., Kbar & $E_{c}, \mathrm{eV}$ & $\Delta E_{g}, \mathrm{eV}$ \\
\hline HF & 3.931 & 2026 & 28.2 & 11.50 \\
DFT-LDA & 3.871 & 1984 & 39.2 & 2.71 \\
DFT-B3LYP & 3.947 & 1791 & 30.7 & 4.38 \\
DFT-PW91 & 3.944 & 1725 & 33.9 & 2.59 \\
Experimental & 3.903 & 1830 & 31.7 & 3.2 \\
\hline \hline
\end{tabular}

been used in DFT calculations. Notice that the definition of the hybrid B3LYP functional implemented in the Crystal code is slightly different from that of Gaussian98.

\section{RESULTS}

\section{A. Electronic structure of bulk $\mathrm{SrTiO}_{3}$}

The fundamental properties of bulk $\mathrm{SrTiO}_{3}$, density of states, lattice parameter $\left(a_{0}\right)$, bulk modulus, cohesive energy $\left(E_{c}\right)$, and energy gap $\left(\Delta E_{g}\right)$, have been computed using various methods with the Crystal code. The bottom of the lowest conduction band lines at the $\Gamma$ point, but is close in energy to the $X$ point. The system presents an optical band gap at the $\Gamma$ point of $4.38 \mathrm{eV}$ (B3LYP result), to be compared with the experimental band gap of $3.2 \mathrm{eV}^{2}$ A much larger energy gap is found at the HF level, $12.50 \mathrm{eV}$, while the PW91 and the LDA calculations give a band gap of 2.6-2.7 $\mathrm{eV}$, Table I. This is in line with the general tendency of HF to give very high energy gaps and of DFT to slightly underestimate this quantity. ${ }^{20,34}$ The mixing of the HF exchange with the DFT one as in the B3LYP method results in a band gap which is too high by about $1 \mathrm{eV}$.

A crude estimate of the band gap can be obtained also from cluster calculations. In this case the gap is defined as the energy difference between the highest occupied and the lowest unoccupied energy levels in the nondefective cluster. The results are qualitatively similar to those obtained with periodic calculations, indicating a gap of $7.9 \mathrm{eV}$ in $\mathrm{HF}$ and of $2.8 \mathrm{eV}$ in DFT-B3LYP $\left(\mathrm{Sr}_{12} \mathrm{Ti}_{12} \mathrm{O}_{36}\right.$ cluster with basis set A). The absolute values, however, are considerably lower than those of the periodic approach.

An important aspect of the electronic structure is the amount of charge separation in the nondefective material. With the large basis set B, the Mulliken analysis carried out on the HF cluster wave function indicates a charge on $\mathrm{Ti}$ of $+2.7, \mathrm{Sr}+1.9$ (average), and $\mathrm{O}-1.5$ (average); at the B3LYP level the values are $\mathrm{Ti}+2.3, \mathrm{Sr}+1.9$ (average), and $\mathrm{O}-1.4$ (average). The Crystal B3LYP results are similar, +2.6 for $\mathrm{Ti},+1.9$ for $\mathrm{Sr}$ and -1.5 for $\mathrm{O}$. Thus, while the formal charge on the $\mathrm{Sr}$ ion is close to the nominal one, for the $\mathrm{Ti}$ and the $\mathrm{O}$ ions there is a significant deviation from the nominal ionicity. A similar result has been reported by other authors. ${ }^{6,43}$

The density of states of $\mathrm{SrTiO}_{3}$, computed at the B3LYP level, has been projected on the atomic orbitals of the constituent atoms. It turns out that the Sr levels do not contribute to the valence band nor to the conduction band, in agreement with the nearly full ionic character of the $\mathrm{Sr}$ ions. The valence band is dominated by the $\mathrm{O} 2 p$ levels with same admixture of the Ti $3 d$ states (partial covalent character). The bottom of the conduction band is dominated by empty Ti $3 d$ states, with very small contributions from the $\mathrm{O}$ ligands.

The lattice constant, the bulk modulus and the cohesive energy of $\mathrm{SrTiO}_{3}$ are given in Table I. The HF method gives a good lattice constant, a large bulk modulus, and, not surprisingly, a too small $E_{c}$; the LDA approach is slightly better for the bulk modulus while largely overestimates the cohesive energy as expected from the self-interaction energy problem associated with LDA. Gradient-corrected functionals provide a considerable improvement. This is true in particular for the DFT-B3LYP approach, which provides the best overall agreement, Table I. The GGA-PW91 approach fails in giving an accurate description of the bulk modulus.

\section{B. The $\mathrm{O}$ vacancy in $\mathrm{SrTiO}_{3}$ : cluster calculations}

The removal of an $\mathrm{O}$ atom from the $\mathrm{Sr}_{6} \mathrm{Ti}_{6} \mathrm{O}_{18}$ and the $\mathrm{Sr}_{12} \mathrm{Ti}_{12} \mathrm{O}_{36}$ clusters results in the formation of an impurity state in the band gap. At the $\mathrm{HF}$ level the state is about $5 \mathrm{eV}$ above the top of the valence band (cluster HOMO) and only $2.7 \mathrm{eV}$ below the conduction band edge (cluster LUMO); at the DFT-B3LYP level the state is $2.4 \mathrm{eV}$ above the top of the valence band, hence very close to the empty states contributing to the conduction band which lies at about $0.4 \mathrm{eV}$ above it. Two possible solutions have been considered for the electronic ground state, an open shell triplet and a closed shell singlet. In the triplet state the electrons involved in the bonding with the missing $\mathrm{O}$ atom (or in an ionic picture formally associated with the $\mathrm{O}^{2-}$ lattice ion) can redistribute and localize into the $3 d$ levels of the nearest $\mathrm{Ti}$ atoms; the electrons can occupy $3 d\left(z^{2}\right)-e_{g}$ type levels pointing along the Ti-Vac-Ti axis, assuming that the $z$ axis corresponds to the Ti-Vac-Ti direction, or enter in one of $3 d-t_{2 g}$ levels of the $\mathrm{Ti}$ atom. For the singlet state there are at least two possible physical situations: (1) the two electrons associated with the vacancy are trapped by the Madelung potential in the cavity giving rise to an electron distribution which is reminiscent of the F centers in ionic crystals; (2) the two electrons are localized on the $3 d\left(z^{2}\right)-e_{g}$ orbitals on two different $\mathrm{Ti}$ atoms but these orbitals are partially overlapping so that the two electrons are singlet-coupled. At this point one has to add that a third possibility exists, namely an openshell singlet which differs from the triplet solution only in the spin coupling; i.e., the two possible spin coupling states arising from well separated unpaired electrons. This openshell singlet will have an energy very close to that of the triplet state. In the following we will provide evidence that the electronic ground state of the oxygen vacancy is nonmagnetic and, hence, the open-shell singlet does not need to be further considered.

In the cluster calculations the triplet is lower in energy in most but not in all cases. In fact, the singlet-triplet energy separation, $\Delta E(T-S)$, depends critically on the size of the cluster and on the completeness of the basis set. With the smaller $\mathrm{Sr}_{6} \mathrm{Ti}_{6} \mathrm{O}_{18}$ cluster $\Delta E(T-S)$ decreases from $2.41 \mathrm{eV}$ with basis A to $1.48 \mathrm{eV}$ with basis $\mathrm{B}$ (a positive $\Delta E$ corre- 
TABLE II. Properties of an isolated $\mathrm{O}$ vacancy in $\mathrm{SrTiO}_{3}$ as derived from embedded cluster calculations.

\begin{tabular}{|c|c|c|c|c|c|c|}
\hline \multirow[b]{3}{*}{ Basis set } & \multicolumn{4}{|c|}{$\mathrm{Sr}_{6} \mathrm{Ti}_{6} \mathrm{O}_{18}$} & \multicolumn{2}{|c|}{$\mathrm{Sr}_{12} \mathrm{Ti}_{12} \mathrm{O}_{36}$} \\
\hline & \multicolumn{2}{|c|}{$\mathrm{HF}$} & \multicolumn{2}{|c|}{ B3LYP } & \multirow{2}{*}{$\frac{\mathrm{HF}}{A}$} & \multirow{2}{*}{$\frac{\text { B3LYP }}{A}$} \\
\hline & $A$ & $B$ & $A$ & $B$ & & \\
\hline$\Delta E_{\text {gap }}, \mathrm{eV}^{\mathrm{a}}$ & 9.14 & 8.91 & 1.80 & 1.96 & 7.91 & 2.79 \\
\hline Ground state & triplet & triplet & triplet & singlet & triplet & triplet \\
\hline$\Delta E(T-S), \mathrm{eV}$ & 2.41 & 1.48 & 1.42 & -0.05 & 1.78 & 0.67 \\
\hline$E_{F}, \mathrm{eV}$ & 4.99 & 5.66 & 7.16 & 8.79 & 5.20 & 7.36 \\
\hline
\end{tabular}

${ }^{\mathrm{a} C o m p u t e d ~ a s ~ t h e ~ H O M U-L U M O ~ d i f f e r e n c e ~ o n ~ t h e ~ n o n d e f e c t i v e ~ c l u s t e r . ~}$

sponds to a triplet ground state); the effect is even more pronounced at the DFT level since $\Delta E(T-S)$ is $1.42 \mathrm{eV}$ with basis set $\mathrm{A}$, while with the large basis $\mathrm{B}$ the situation is reversed and the singlet becomes slightly more stable, by $0.05 \mathrm{eV}$, Table II. This clearly shows that by increasing the basis set size, in particular by including diffuse functions, the singlet state becomes more stabilized. This effect is observed also by increasing the size of the cluster. At the HF level, going from $\mathrm{Sr}_{6} \mathrm{Ti}_{6} \mathrm{O}_{18}$ to $\mathrm{Sr}_{12} \mathrm{Ti}_{12} \mathrm{O}_{36} \Delta E(T-S)$ decreases by $0.6 \mathrm{eV}$, Table II; also in this case, the effect is more pronounced at the DFT level, where the reduction of $\Delta E(T-S)$ by doubling the size of the cluster is of $0.75 \mathrm{eV}$. A calculation of the $\mathrm{Sr}_{12} \mathrm{Ti}_{12} \mathrm{O}_{36}$ cluster with the large basis set $\mathrm{B}$ is outside our present computational possibilities, but the trends discussed above strongly suggest that this should result in a singlet ground state.

The formation energy, defined with respect to a free, gasphase $\mathrm{O}$ atom, is $7.16 \mathrm{eV}$ at the B3LYP level with the $\mathrm{Sr}_{6} \mathrm{Ti}_{6} \mathrm{O}_{18}$ cluster, basis $\mathrm{A}$, and of $7.36 \mathrm{eV}$ with the same basis set but with the larger $\mathrm{Sr}_{12} \mathrm{Ti}_{12} \mathrm{O}_{36}$ cluster. This shows that the formation energy is relatively well converged with respect to the cluster size. However, the increase of the basis set from A to $\mathrm{B}$ using the $\mathrm{Sr}_{6} \mathrm{Ti}_{6} \mathrm{O}_{18}$ cluster results in a further increase of $E_{F}$ to $8.8 \mathrm{eV}$, Table II, indicating that this quantity is not fully converged with respect to the basis set size. Therefore, the value of about $9 \mathrm{eV}$ for the formation energy is likely to be slightly underestimated.
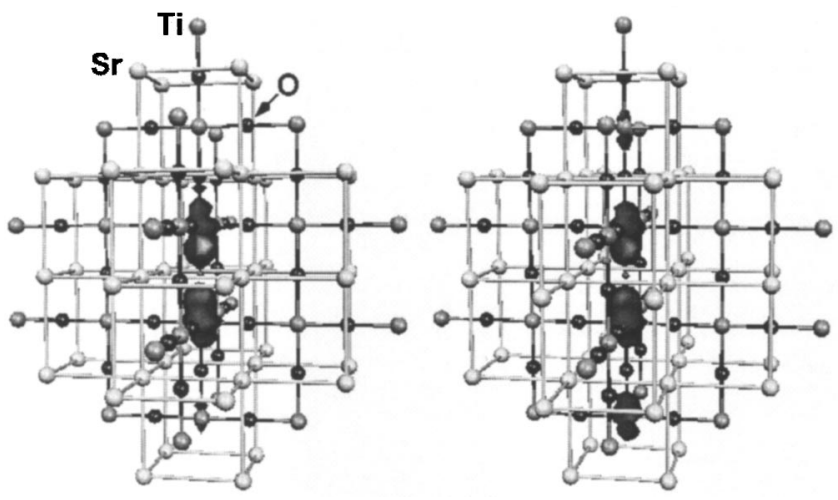

FIG. 3. Spin density plot for the triplet state of an $\mathrm{O}$ vacancy in $\mathrm{SrTiO}_{3}$ obtained with the $\mathrm{Sr}_{12} \mathrm{Ti}_{12} \mathrm{O}_{36}$ cluster and basis A (cutoff 0.01 a.u.). Left: HF. Right: B3LYP. A small sphere at the center indicates the position of the missing $\mathrm{O}$ atom.
The above discussion on the relative stability of the closed-shell singlet and triplet states suggests that the two states have a different degree of localization. In fact, the energy of the singlet is lowered with respect to the triplet by increasing the size of the basis set and of the cluster, as one would expect if the singlet state is more delocalized. In the following we consider and discuss the nature of the two states based on electron density maps and a population analysis.

For the triplet state we report the spin density, $\rho^{\alpha}-\rho^{\beta}$, which provides a direct view of the distribution of the unpaired electrons in the system. Less direct is the visualization of the charge density associated with the singlet state. This could be obtained as the difference of the total density for the nondefective system minus the density of the defective system and that of an $\mathrm{O}$ atom. As an alternative, we report the plot of the molecular orbital (MO) corresponding to the impurity state in the gap associated with the vacancy. In comparing the plots of the singlet and triplet states, Figs. 3 and 4, one has to be aware that different quantities are reported. Still, very useful information about the charge distribution can be obtained in this way.

We restrict the analysis to the $\mathrm{Sr}_{12} \mathrm{Ti}_{12} \mathrm{O}_{36}$ cluster. At the HF level, the triplet state is characterized by two electrons occupying the $3 d\left(z^{2}\right)-e_{g}$ orbitals on the Ti atoms directly above and below the vacancy, Fig. 3(a). There is virtually no residual spin density on the other $\mathrm{Ti}$ atoms, indicating a strong degree of localization. At the B3LYP level the result is similar but we observe a tendency to distribute the unpaired electrons also on the other $\mathrm{Ti}$ atoms along the Ti-Vac-Ti axis, Fig. 3(b). As a result, $84 \%$ of the spin density is around the vacancy, and only the remaining $16 \%$ is delocalized over the $\mathrm{Ti}$ atoms of the cluster, Table III.

For the singlet state, Fig. 4, we concentrate our attention on the shape of the doubly occupied MO in the band gap. At the HF level we observe a considerable electron density at the center of the vacancy, Fig. 4(a); the effect is found also at the DFT-B3LYP level, Fig. 4(b). The contour of the MO is

TABLE III. Spin distribution in an oxygen vacancy in the bulk $\mathrm{SrTiO}_{3}$ (triplet state and B3LYP results).

\begin{tabular}{cccc}
\hline \hline & $\begin{array}{c}\mathrm{Sr}_{12} \mathrm{Ti}_{12} \mathrm{O}_{36} \\
\text { cluster }\end{array}$ & $\begin{array}{c}40 \text { atoms } \\
\text { supercell }\end{array}$ & $\begin{array}{c}80 \text { atoms } \\
\text { supercell }\end{array}$ \\
\hline $\mathrm{Ti}_{1,2}$ & 0.84 & 0.79 & 0.83 \\
$\mathrm{Ti}$ (average) & 0.05 & 0.08 & 0.02 \\
\hline \hline
\end{tabular}



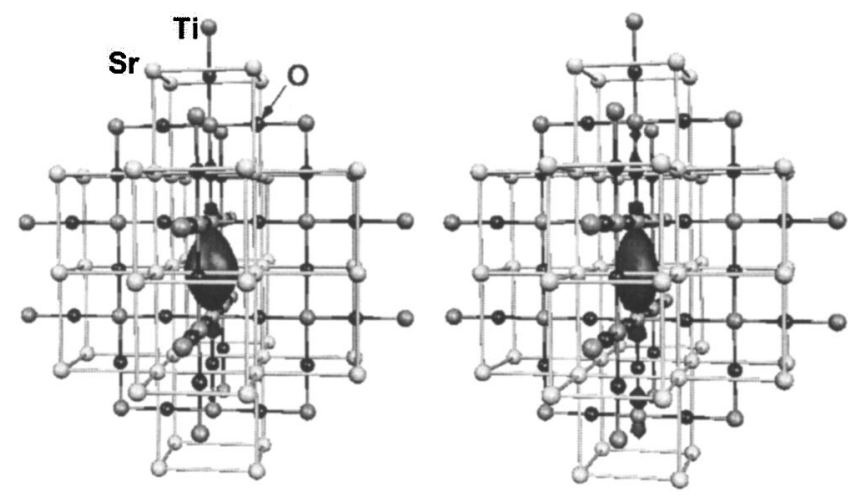

FIG. 4. Plot of the doubly occupied MO corresponding to the impurity state in the band gap associated with an $\mathrm{O}$ vacancy in $\mathrm{SrTiO}_{3}$ obtained with the $\mathrm{Sr}_{12} \mathrm{Ti}_{12} \mathrm{O}_{36}$ cluster and basis A (cutoff 0.06 a.u.). Left: HF. Right: B3LYP.

due to the overlap of the $3 d\left(z^{2}\right)-e_{g}$ orbitals on the two Ti atoms with the functions centered in the vacancy. In this respect, the charge distribution associated with the $\mathrm{O}$ vacancy is quite different from that of a classical $\mathrm{F}$ center in alkali halides or alkaline-earth oxides. In this latter case in fact the maximum of the electron density is at the center of the vacancy and the distribution is isotropic. In the case of $\mathrm{SrTiO}_{3}$, on the contrary, the maximum of the electron density is near the Ti atoms and the charge distribution is elongated along the direction of the Ti-Vac-Ti axis. This will be clearer in another view of the charge density obtained with the periodic calculation and discussed in the next section. The charge distribution is consistent with the position of the defect level which is close to the conduction band, in particular at the B3LYP level, indicating a substantial contribution from the Ti $3 d$ states.

To summarize this section, the cluster calculations show that the removal of an $\mathrm{O}$ atom from the lattice has an high energy cost ( $9 \mathrm{eV}$ or more). Two electrons are transferred from the valence of the central $\mathrm{O}$ atom to the empty $3 d$ states of the Ti nearest neighbors; the wave functions of the two electrons do overlap to a certain extent so that a competition is found for the two states where the spin are singlet or triplet coupled. By extending the size of both the QM region and of the basis set, there is a clear tendency to favor the singlet state, more delocalized, with respect to the triplet one.

\section{The neutral $\mathrm{O}$ vacancy in $\mathrm{SrTiO}_{3}$ : Periodic calculations}

To describe the formation of an $\mathrm{O}$ vacancy in the bulk of $\mathrm{SrTiO}_{3}$ we have used two supercells. The first one, containing 40 atoms, Fig. 2, corresponds to a rather high density of defects, $\mathrm{SrTiO}_{3-\delta}$ with $\delta=0.125$. One every two oxygen atoms along the Ti-O-Ti $c$ axis has been removed. This could result in the interaction of the wave functions of the individual vacancies. In order to remove this problem, the size of the cell has been doubled along the $c$ axis (80-atoms supercell). In this way the Ti atoms nearest to the vacancy are properly coordinated and sufficiently distant from the next defect. With the 40-atoms unit cell the electronic structure of the vacancy has been determined at four theoretical levels: HF, LDA, B3LYP and PW91. For the larger 80-atoms cell we restricted the analysis to the HF and B3LYP methods.

The DOS curves, Fig. 5, show that by creating the vacancy, a new occupied state appears in the gap, at $3.4 \mathrm{eV}$ above the top of the valence band and $0.8 \mathrm{eV}$ below the bottom of the conduction band (singlet state). This is consistent with experimental estimates which indicated an ionization energy for the neutral oxygen vacancy of the order of $0.4 \mathrm{eV}^{44}$ and with optical measurements which suggest the presence vacancies-induced states slightly below the conduction band edge. ${ }^{45}$ A second empty state is very close to the first one, Fig. 5. It should be mentioned however that the interpretation of these experiments is far from being unambiguous: in fact, along with $\mathrm{F}$ centers many other defects or defect complexes can contribute to the observed states. Other experiments indicate that F-type centers have optical absorptions around $2.7 \mathrm{eV}$, thus suggesting that the $\mathrm{F}$ center corresponds to a deep state in the gap. ${ }^{36}$

These levels have a significant contribution from the $\mathrm{Ti}$ $3 d$ orbitals but are mixed-in with the orbitals placed at the center of the vacancy. The two levels can be seen as a bond-

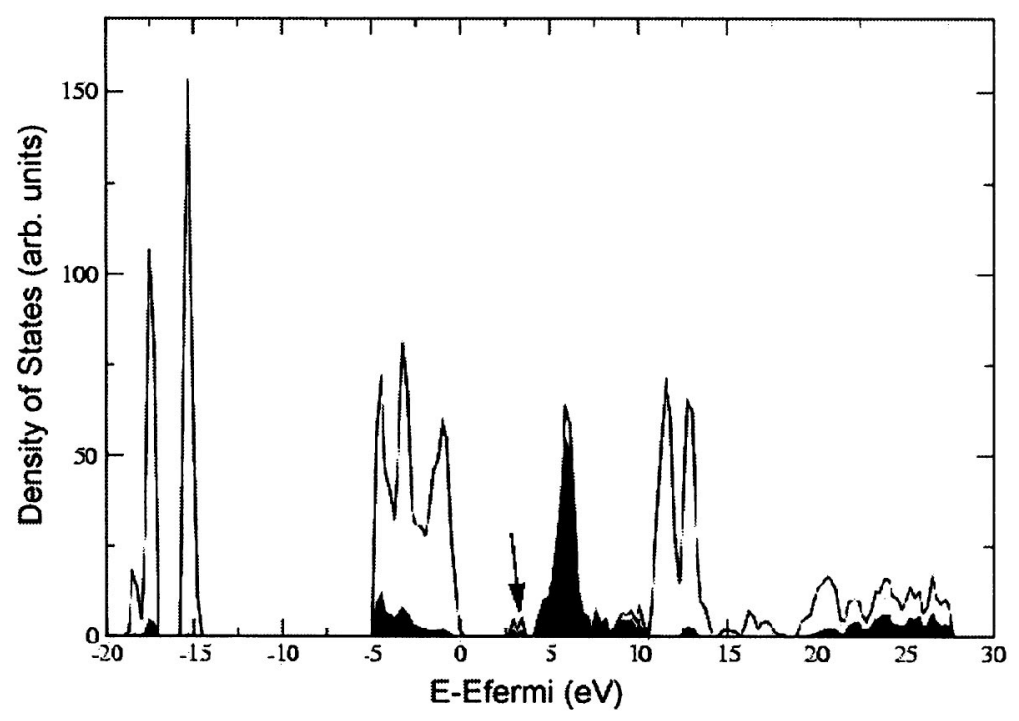

FIG. 5. Total and Ti-projected (dark) density of states (DOS) for an $\mathrm{O}$ vacancy in $\mathrm{SrTiO}_{3}$ (singlet state) as obtained at the B3LYP level on a 80 atoms supercell. The contribution of the vacancy states in the gap are shown by an arrow. 
TABLE IV. Properties of an isolated $\mathrm{O}$ vacancy in $\mathrm{SrTiO}_{3}$ as derived from periodic calculations.

\begin{tabular}{|c|c|c|c|c|c|c|}
\hline & \multicolumn{4}{|c|}{$\mathrm{Sr}_{8} \mathrm{Ti}_{8} \mathrm{O}_{24}$ (40 atoms) } & \multicolumn{2}{|c|}{$\mathrm{Sr}_{16} \mathrm{Ti}_{16} \mathrm{O}_{48}$ (80 atoms $)$} \\
\hline & $\mathrm{HF}$ & LDA & B3LYP & PW91 & $\mathrm{HF}$ & B3LYP \\
\hline Ground state & triplet & singlet & singlet & singlet & singlet $^{\mathrm{a}}$ & singlet \\
\hline$\Delta E(T-S), \mathrm{eV}$ & 0.13 & -0.28 & -0.53 & -0.27 & -1.03 & -0.63 \\
\hline$E_{F}, \mathrm{eV}$ & 7.73 & 10.90 & 9.94 & 9.75 & 7.79 & 9.42 \\
\hline
\end{tabular}

${ }^{\mathrm{a}}$ In this case we were unable to converge the calculations on the $3 d\left(z^{2}\right)-e_{g}$ which is likely to be the lowest triplet configuration; see text.

ing and antibonding combinations of $3 d\left(z^{2}\right)$ orbitals on the two Ti atoms directly above and below the vacancy. These states are separated from the conduction band, showing that the defect states have a distinct character from those contributing to the conduction band (mainly Ti $3 d$ empty levels).

The first question of interest is the nature of the ground state. With the 40-atoms supercell we found that the triplet configuration is slightly preferred (by $0.13 \mathrm{eV}$ ) only at the HF level. At the DFT level the singlet state is always lower in energy, with energy differences between triplet and singlet that go from 0.3 to $0.5 \mathrm{eV}$, Table IV. The preference for a triplet state in $\mathrm{HF}$ is not too surprising if we consider the already mentioned general tendency of this method to localize spins and favor open shell structures. The preference for a closed shell singlet ground state is confirmed by the calculations done with the larger, 80-atoms supercell. Here the singlet state is preferred not only in B3LYP, but also at the HF level. The HF calculation has not converged on the lowest triplet configuration but on a state where the unpaired electrons are localized on a $t_{2 g}$ combination of $3 d$ orbitals. Unfortunately we were unable to obtain proper convergence on the $3 d\left(z^{2}\right)-e_{g}$ triplet state in HF which is the lowest triplet in the other calculations (in general we observed a very slow convergence of the periodic calculations for the open shell solutions).

From the periodic calculations it emerges quite clearly that the singlet state is the most stable one; this result is obtained at the DFT level with various exchange-correlation functionals, even including a contribution from the exact HF exchange as in B3LYP. We have seen above that with cluster models the singlet is also the most stable state provided that sufficiently large clusters and basis sets are used. The fact that the closed-shell singlet state is lower than the open-shell triplet state is a strong proof of the non-magnetic character of the electronic ground state. In fact, for a magnetic state one will have the triplet (ferromagnetic) and open-shell singlet (antiferromagnetic) states near degenerate and well below the close-shell singlet. Having established that the nature of the ground state is nonmagnetic, we move now to the analysis of the charge distribution in the vacancy, Table IV.

We begin from the analysis of the charge distribution in the singlet ground state of the $\mathrm{O}$ vacancy. We have plotted the charge density of the system for an energy range corresponding to the electronic state (Kohn-Sham eigenvalue) associated with the vacancy in the gap (see Fig. 5); in this energy range only one doubly occupied level is present and the charge density integrates to two electrons. The plots, Fig.
6 , show that the two electrons are largely localized on the $\mathrm{Ti}$ $3 d\left(z^{2}\right)-e_{g}$ orbitals with substantial extension of the electron cloud towards the center of the vacancy. This is true at both HF and B3LYP levels, Fig. 6. It is important to note that the charge distribution obtained with a periodic approach is qualitatively similar to that obtained from the cluster calculations, see Fig. 4. The chemical reduction of the Ti ions adjacent to the vacancy from $\mathrm{Ti}^{4+}$ to $\mathrm{Ti}^{3+}$ should result in a shift of the Ti core levels.

The situation is somewhat more complex when we analyze the spin distribution in the triplet state. We restrict the analysis to the B3LYP results which give the same lowest triplet configuration with the 40 -atoms and the 80 -atoms supercell. In B3LYP the spin is partially delocalized over many Ti centers, although the largest contribution is from the two $\mathrm{Ti}$ atoms nearest to the vacancy. A semiquantitative evaluation of the degree of delocalization is provided by the results of the spin population which show that about $80 \%$ of the unpaired electrons is on the Ti atoms above and below the vacancy, while the rest is more or less equally divided among the remaining Ti atoms of the supercell, Table III. Also this result is qualitatively similar to that obtained with cluster models, indicating a substantial equivalence of the two physical situations.

Finally we consider the formation energy of the oxygen vacancy. In this respect the most accurate values are those obtained using gradient corrected B3LYP and PW91 functionals. The HF and the LDA values obtained with the 40atoms supercell, 7.73 and $10.90 \mathrm{eV}$, respectively, are lower and upper bounds to the actual energy cost. Both the B3LYP and the PW91 methods predict a formation energy close to
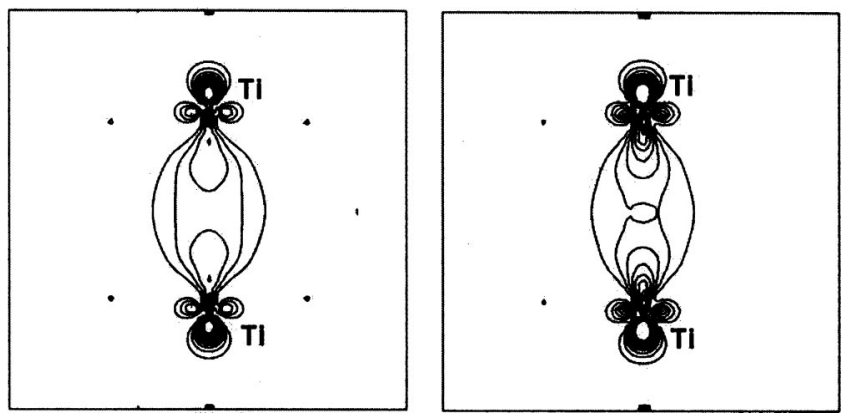

FIG. 6. Charge density plot of the doubly occupied impurity state in the gap of the material associated with the oxygen vacancy (singlet ground state) in the plane containing the Ti-Vac-Ti axis. Left: HF. Right: B3LYP. 
$10 \mathrm{eV}$, Table IV. Our best estimate obtained at the B3LYP level with the 80 -atoms supercell is $9.4 \mathrm{eV}$. This is very similar to that estimated for an $\mathrm{O}$ vacancy in the bulk of $\mathrm{MgO}, 9.5 \mathrm{eV}^{46}$ It is interesting to note that the formation energy computed with a periodic approach, $9.4 \mathrm{eV}$, is about $0.6 \mathrm{eV}$ larger than that obtained from cluster calculations $(8.8$ $\mathrm{eV}$ with basis set $\mathrm{B}$ and the $\mathrm{Sr}_{6} \mathrm{Ti}_{6} \mathrm{O}_{18}$ cluster, Table II). This is probably due to the incompleteness of the basis set in the cluster calculations but could also arise in part from the electrostatic potential at the vacancy site provided by the embedding in formal point charges.

\section{CONCLUSIONS}

The nature of the oxygen vacancy in $\mathrm{SrTiO}_{3}$ has been addressed previously, ${ }^{4-12}$ although only few studies have been performed at an $a b$ initio level..$^{6,9,10,11}$ In this paper we have considered for the first time the competition between magnetic and non-magnetic coupling of the two extra electrons associated with the vacancy. In order to do this, various methods and computational approaches have been adopted. In fact, it has been shown that the use of pure DFT methods may result in misleading results when spin localization processes are involved. ${ }^{22}$ The main features of our study can be summarized as follows.

(1) Different kinds of bonds exist in $\mathrm{SrTiO}_{3}$. The Sr-O interactions are very ionic, as for binary alkaline-earth oxides, while the Ti-O bonding has a mixed ionic-covalent character. This leads in principle to a competition between the tendency to trap the electrons associated with the missing oxygen in the vacancy ( $\mathrm{F}$ center) or to localize them on the $3 d$ levels of the transition metal ion. The results show that the electrons are not localized in the vacancy. In this respect, the notation of $\mathrm{F}$ center for an $\mathrm{O}$ vacancy in $\mathrm{SrTiO}_{3}$ is not fully justified. The creation of a neutral $\mathrm{O}$ vacancy results in new states in the band gap, below the conduction band. This is consistent with previous theoretical studies ${ }^{6}$ and with experimental estimates which indicated a very small ionization energy for the oxygen vacancy, of the order of $0.4 \mathrm{eV}^{44}$ Optical measurements suggest that the presence of oxygen vacancies induce states slightly below the conduction band edge, ${ }^{45}$ although these assignments are not unambiguous. ${ }^{36}$

(2) These states are mainly composed of $3 d\left(z^{2}\right)$ levels of the two Ti atoms directly above and below the vacancy. The states are considerably mixed in with the $s$-type orbitals at the center of the cavity, giving rise to a substantial overlap of the wave functions. The localization of the electrons on $\mathrm{Ti}$ is consistent with photoemission studies on the surface of $\mathrm{SrTiO}_{3}$ which indicate the formation of $\mathrm{Ti}^{3+}$ ions when surface defects are created by $\mathrm{Ar}^{+}$-ion bombardment. ${ }^{47,48}$

(3) The ground state of the system is nonmagnetic since the two electrons are singlet coupled (singlet closed-shell ground state). While the exact singlet-triplet separation may depend on the size of the supercell used for the calculations, the qualitative result is not, suggesting that this conclusion is not dependent on this aspect of the calculation. The triplet solution is slightly lower in energy only at the HF level. A singlet ground state is what one expects for $\mathrm{F}$ centers in ionic crystals, but differs from what has been found in $\mathrm{TiO}_{2}$ where the formation of an $\mathrm{O}$ vacancy results in a triplet ground state with spins localized on two different Ti atoms. ${ }^{24}$ In $\mathrm{SrTiO}_{3}$ the singlet ground state is the result of the coupling of the two electrons which occupy $3 d\left(z^{2}\right)$ levels on the two $\mathrm{Ti}$ atoms. The charge distribution is similar to that found for the same kind of defect in other perovskites like $\mathrm{PbTiO}_{3},{ }^{10}$ $\mathrm{BaTiO}_{3},{ }^{11}$ and $\mathrm{KNbO}_{3}{ }^{35}$

(4) The formation energy of the isolated vacancy, computed with respect to an $\mathrm{O}\left({ }^{3} \mathrm{P}\right)$ atom, is close to $10 \mathrm{eV}(9.4$ $\mathrm{eV}$ is our best estimate). Since the strength of the $\mathrm{O}_{2}$ bond is correctly reproduced in $\mathrm{B} 3 \mathrm{LYP}, 5.2 \mathrm{eV}$, this corresponds to a formation energy of $6.8 \mathrm{eV}$ with respect to $1 / 2 \mathrm{O}_{2}$.

\section{ACKNOWLEDGMENTS}

The work has been supported by the Italian Ministry of University and Research (MIUR) through a Cofin project. Computer time was provided by the Center de Supercomputació de Catalunya, CESCA, and the Center Europeu de Paral.lelisme de Barcelona, CEPBA through the European Community Improving Human Potential (IHP) program under contract HPRI-CT-1999-00071 held by the CESCA/ CEBPA; CRYSTAL calculations have been carried out on the SP3 parallel machine of the CEPBA-IBM-ResearchInstitute of Barcelona. D.R. is grateful to the European Community and to the Italian-Spain Integrated Action for financing his stay in Barcelona.
*Email address: gianfranco.pacchioni@unimib.it

${ }^{1}$ R. J. D. Tilley, Principles and Applications of Chemical Defects (Stanley Thornes, Cheltenham, 1998).

${ }^{2}$ T. A. Noland, Phys. Rev. 94, 724 (1954).

${ }^{3}$ J. F. Schooley, W. R. Hosler, and M. L. Cohen, Phys. Rev. Lett. 12, 474 (1964).

${ }^{4}$ M. Tsukada, C. Satoko, and H. Adachi, J. Phys. Soc. Jpn. 48, 200 (1980).

${ }^{5}$ M. O. Selme and P. Pecheur, J. Phys. C 16, 2559 (1983).

${ }^{6}$ S. Kimura, J. Yamauchi, M. Tsukada, and S. Watanabe, Phys. Rev. B 51, 11049 (1995).

${ }^{7}$ M. A. Burin, S. A. Prosandeyev, I. I. Gegusin, and I. M. Tennenboum, Radiat. Eff. Defects Solids 134, 75 (1995).

${ }^{8}$ S. A. Prosandeyev, A. V. Fisenko, A. I. Riabachinski, I. O. Os- ipenko, I. P. Raevski, and N. Safontseva, J. Phys.: Condens. Matter 8, 6705 (1996).

${ }^{9}$ N. Shanthi and D. D. Sarma, Phys. Rev. B 57, 2153 (1998).

${ }^{10}$ C. H. Park and D. J. Chadi, Phys. Rev. B 57, R13961 (1998).

${ }^{11}$ H. Donnerberg and A. Birkholz, J. Phys.: Condens. Matter 12, 8239 (2000).

${ }^{12}$ A. Stashans and F. Vargas, Mater. Lett. 50, 145 (2001).

${ }^{13}$ R. Astala and P. D. Bristowe, Comput. Mater. Sci. 22, 81 (2001).

${ }^{14}$ C. A. Coulson, Valence (Clarendon, Oxford, 1952).

${ }^{15}$ L. Thomas, Proc. Cambridge Philos. Soc. 23, 542 (1927); E. Fermi, Z. Phys. 49, 550 (1928).

${ }^{16}$ N. F. Mott and M. J. Littleton, Trans. Faraday Soc. 34, 485 (1938).

${ }^{17}$ W. Kohn and L. J. Sham, Phys. Rev. 140, A1133 (1965). 
${ }^{18}$ R. L. Martin and F. Illas, Phys. Rev. Lett. 79, 1539 (1997).

${ }^{19}$ F. Illas and R. L. Martin, J. Chem. Phys. 108, 2519 (1998).

${ }^{20}$ I. P. R. Moreira, F. Illas, and R. L. Martin, Phys. Rev. B 65, 155102 (2002).

${ }^{21}$ R. H. D. Nuttall and J. A. Weil, Can. J. Phys. 59, 1696 (1981).

${ }^{22}$ G. Pacchioni, F. Frigoli, D. Ricci, and J. A. Weil, Phys. Rev. B 63, 054102 (2001).

${ }^{23}$ G. Pacchioni and G. Ieranò, Phys. Rev. Lett. 79, 753 (1997).

${ }^{24}$ T. Bredow and G. Pacchioni, Chem. Phys. Lett. 355, 417 (2002).

${ }^{25}$ W. C. Mackrodt, E.-A. Simson, and N. M. Harrison, Surf. Sci. 384, 192 (1997).

${ }^{26}$ U. Diebold, Surf. Sci. Rep. 48, 53 (2003).

${ }^{27}$ W. Stevens, H. Bach, and J. Krauss, J. Chem. Phys. 81, 6026 (1984).

${ }^{28}$ R. Ditchfield, W. J. Hehre, and J. A. Pople, J. Chem. Phys. 54, 724 (1971).

${ }^{29}$ P. J. Hay and W. R. Wadt, J. Chem. Phys. 82, 284 (1985); 82, 299 (1985).

${ }^{30}$ F. Illas and G. Pacchioni, J. Chem. Phys. 108, 7835 (1998).

${ }^{31}$ A. D. Becke, J. Chem. Phys. 98, 5648 (1993).

${ }^{32}$ C. Lee, W. Yang, and R. G. Parr, Phys. Rev. B 37, 785 (1988).

${ }^{33}$ W. Kock and M. C. Holthausen, A Chemist's Guide to Density Functional Theory (Wiley-VCH, Weinheim, 2002).

${ }^{34}$ J. Muscat, A. Wander, and N. M. Harrison, Chem. Phys. Lett. 342, 397 (2001).

${ }^{35}$ R. I. Eglitis, N. E. Christensen, E. A. Kotomin, A. V. Postnikov, and G. Borstel, Phys. Rev. B 56, 8599 (1997).
${ }^{36}$ E. Kotomin and A. Popov, Nucl. Instrum. Methods Phys. Res. B 141, 1 (1998).

${ }^{37}$ W. Gong, H. Yun, Y. B. Ning, J. E. Greedan, W. R. Datans, and C. V. Stager, J. Solid State Chem. 90, 320 (1991).

${ }^{38}$ M. J. Frish et al., Gaussian98 (Gaussian Inc., Pittsburgh, PA, 1998).

${ }^{39}$ R. A. Evarestov, S. Piskunov, E. A. Kotomin, and G. Borstel, Phys. Rev. B 67, 064101 (2003).

${ }^{40}$ V. R. Saunders, R. Dovesi, C. Roetti, M. Causà, N. M. Harrison, R. Orlando, and C. M. Zicovich-Wilson, CRYSTAL 98 User's Manual, University of Torino, Torino, 1998.

${ }^{41}$ M. P. Habas, R. Dovesi, A. Lichanot, J. Phys.: Condens. Matter 10, 6897 (1998).

${ }^{42}$ P. Reinhart, B. Hess, and A. Causà, Int. J. Quantum Chem. 58, 296 (1996).

${ }^{43}$ E. Heiftets, R. I. Eglitis, E. A. Kotomin, J. Maier, and G. Borstel, Surf. Sci. 513, 211 (2002).

${ }^{44}$ I. P. Raevski, S. M. Maksimov, A. V. Fisenko, S. A. Prosandeyev, I. A. Osipenko, and P. F. Tarasenko, J. Phys.: Condens. Matter 10, 8015 (1998).

${ }^{45}$ C. Lee, J. Destry, and J. L. Brebner, Phys. Rev. B 11, 2299 (1975).

${ }^{46}$ L. Giordano, J. Goniakowski, and G. Pacchioni, Phys. Rev. B 64, 075417 (2001).

${ }^{47}$ V. E. Henrich, G. Dresselhaus, and H. J. Zeiger, Phys. Rev. B 17, 4908 (1978).

${ }^{48}$ Y. Adachi, S. Kohiki, K. Wagatsuma, and M. Oku, Appl. Surf. Sci. 143, 272 (1999). 